\title{
PROCESOS TRANSACCIONALES Y DESARROLLO AUTOGESTIVO EN SALUD Y ATENCIÓN BUCAL ENTRE LOS GRUPOS (QOM) TOBAS ASENTADOS EN LOS PUMITAS (ROSARIO), ARGENTINA.
}

\author{
María Susana AZCONA
}

\section{Resumen}

El autor analiza críticamente los procesos transaccionales establecidos entre los profesionales de los servicios públicos de salud / Centro Comunitario: QADUOQHQTE (Qom) tobas, asentados en los Pumitas (Rosario) Argentina;referidos al proceso salud/ enfermedad/ atención bucal dentro del contexto de políticas sociales y sanitarias emanadas de jurisdicciones municipales, provinciales y nacionales

Palabras clave: Indígenas toba -Derechos sociales y salud- Servicios OdontológicosDesarrollo autogestivo.

\begin{abstract}
In this paper the author critically analyzes the transactional processes operating between the professionals of public health services and the Community Centre: QADUOQHQTE (Qom) settled in The Pumitas (Rosario) Argentina, Concerning the process of health / disease / oral care, within the context of social and health policies emanating from national, provincial and municipal jurisdictions.
\end{abstract}

keywords: Toba people -Health and social rights - Dental services- self promoted development.

\section{Resumé}

L'auteur fait une analyse critique des processus transactionnels établis entre les professionnels des services publics de la santé et le Centre Communautaire: QADUOQHQTE (Qom), qui habitent à Los Pumitas (Rosario) Argentina, par rapport au processus santé- maladie- attention buccale dans le contexte de politiques sociales et sanitaires émanant de juridictions municipaux, provinciales et nationales

Mots clés : population toba - Droits sociaux et de 1'santé- Services OdontologiquesDéveloppement autogéré.

\section{Introducción}

\footnotetext{
${ }^{1}$ Investigadora del Consejo de Investigaciones de la Universidad Nacional de Rosario, Categ. B Profesora Titular de la Facultad de Odontología de la UNR.
}

Fecha de recepción del artículo: Septiembr 2010

Fecha de evaluación: Octubre 2010 
Los procesos transaccionales establecidos entre los profesionales de los servicios de salud y el Centro Comunitario QADHUOQTE, se ubican en el marco de las reivindicaciones étnicas que vienen realizando los pueblos originarios en los últimos años.

En este sentido, la construcción de la ciudadanía indígena en América Latina es un tema de importancia creciente tanto de la agenda política como de la reflexión académica. Por consiguiente, ubicamos nuestro análisis al interior de tal contexto. Los conceptos de etnicidad, identidad y acción colectiva se constituyen como conceptos clave del presente texto.

En el debate en torno a la construcción de la ciudadanía indígena, resulta imprescindible privilegiar el análisis de los procesos socio-históricos que sirven de marco a las propuestas organizadoras del Estado y a las acciones emprendidas por los propios actores-indígenas en la dinámica por el reconocimiento y la ampliación de derechos socioétnicos específicos, entre los que la identidad cultural se constituye en eje de acción política. El principal precedente inmediato de los procesos de desarrollo de ciudadanía de esta índole debe buscarse en el fenómeno de globalización y sus consecuentes derivaciones en las transformaciones del Estado, del mercado y de la sociedad civil, lo que produjo profundos cambios en la acción colectiva de los pueblos indígenas, en especial en sus mediaciones interactivas con la comunidad, nacional e internacional. Como consecuencia de esto, las estrategias políticas de los movimientos y organizaciones indígenas se estructuran de distintas formas, pero se expresan y simbolizan a partir de códigos compartidos que les imprimen unidad discursiva Bello, A. (2004)

La creciente relevancia de los movimientos de reivindicaciones de los pueblos originarios se pone en evidencia mediante las acciones que vienen realizando diferentes comunidades indígenas a través de sus organizaciones y dirigentes. Estas actividad es no han contado, siempre, con los apoyos gubernamentales en el plano de la participación efectiva de estas comunidades quedando de tal modo restringida la posibilidad de que sus miembros puedan resolver con provecho cuestiones referidas a su organización y disponibilidad financiera de administración de recursos para actividades y emprendimientos locales de economías regionales, salud, educación, etc.

Los pueblos originarios de nuestro país, entre ellos los Tobas, se ubican entre los segmentos sociales más castigados de la economía argentina, dando continuidad a una matriz de desvalorización y exclusión que ha operado como constante en la historia nacional.

La identidad étnica se elabora como proceso dinámico que se va modificando de acuerdo a las diferentes políticas que establece el Estado Nacional. En los momentos de mayor represión, las identidades se repliegan, y se expanden en los de mayor tolerancia y participación. A partir de 1994, con la reforma de la Constitución Nacional, se incorporan derechos que les reconocen a los pueblos aborigenes el carácter jurídico de pueblos originarios. Destacando que son comunidades preexistentes a la formación del Estado Nacional. Se les reconoce el derecho a la propiedad de la tierra en forma comunitaria, A ser consultados y a participar en la administración y gestión de los recursos naturales localizados en sus tierras, y a la educación intercultural.

Las reivindicaciones etnopolíticas movilizadas por las organizaciones indígenas facilitó la posibilidad de una revisión crítica de las prácticas políticas asociadas a las formas de convivencias y representación política en el seno de las democracias latinoamericanas. Las autonomías constituidas en complejos procesos de acciones de afirmación de las identidades étnicas, se expresan a través de los derechos territoriales, primero, la necesidad de la educación 
bilingüe e intercultural (los derechos lingüísticos) y la generación y organización de movimientos indígenas, después. Vázquez, H. (2002).

Desde una perspectiva que dista de las que impulsan un mestizaje cultural, y también de aquellas que consideran la identidad como inmutable; aceptamos la necesidad de gestar nuevas prácticas de interacción social (nterculturalidad) en la que la necesaria cohesión social se fundamente en la constitución de una ciudadanía basada en un democracia política y social, pero también, como lo destaca Margot Bigot, lingüística , cultural, y étnica. Bigot, M. (2002).

\section{Reflexiones al interior del campo de la salud y de la salud odontológica.}

Sostenemos la necesidad de desarrollar políticas públicas que incluyan los derechos sociales y civiles diferenciados de los pueblos indígenas en materia de salud. Al respecto, es necesario vincular al debate sobre el proceso de salud / enfermedad / atención los aspectos políticos y éticos con relación a las estrategias de participación comunitaria. De tal modo, que los estudios sobre la salud indígena no sólo enfaticen la heterogeneidad, emergencia y dinámica, de la cultura, sino también las interacciones sociales que caracterizan el contexto interétnico, intercultural e intermédico.

La construcción de políticas públicas y la elaboración y ejecución de intervenciones sociales a través de proyectos para los pueblos indígenas son aspectos cruciales.

Se destacan como temas relevantes de las relaciones entre los pueblos indígenas y el Estado, los siguientes: las ofertas financieras a los pueblos indígenas, la participación indígena, los mecanismos de gestión de las políticas públicas, la participación de los diversos actores en los procesos de ejecución de las acciones de estas políticas, la gestión de redes establecidas y los procesos socioculturales y políticos vividos durante el diseño, implementación y ejecución de los proyectos direccionados a las poblaciones indígenas.

En la elaboración de estrategias políticas, económicas, sociales y culturales por parte de los grupos indígenas para fortalecer las actividades que contribuyan a mantener la transmisión del patrimonio cultural de la comunidad, y el mejoramiento de la calidad de vida, se constata una importante tensión que ha movilizado algunos debates en torno al etnodesarrollo / desarrollo autogestivo. Se trata de dos fuerzas en constante tensión en la implementación de este proceso: la construcción política de la autonomía cultural frente al estado nacional / provincial / municipal y las distintas formas de operar al integrarse con las estructuras de la economía nacional e internacional en sus negociaciones.

Desde las últimas décadas, estas poblaciones plantean distintas demandas a las sociedades y gobiernos para potenciar su autodesarrollo, recuperando protagonismo, fomentando prácticas ancestrales, sin dejar de lado los medios tecnológicos que consideran valiosos para el mejoramiento de su situación comunitaria y la promoción de su cultura.

No existen registros sobre el estado de salud de las mismas. Estas comunidades son prácticamente marginadas de cualquier tipo de relevamiento de datos epidemiológicos en general y en particular bucodentales.

Asimismo son escasas o nulas las investigaciones y estudios sobre estas comunidades. Existe pues no solo un desconocimiento epidemiológico sino, lo más importante, es que se desconocen los sistemas de valores y de representaciones sociales de estas etnias sobre el proceso salud / enfermedad / atención, específicamente del componente bucal. 
La etnoepidemiología realiza aportes muy considerables a nuestro campo de conocimiento. Esta disciplina, no se define solamente por utilizar métodos circunscriptos a la epidemiología clásica propia de investigaciones de estudios interculturales en salud. Sino por su adopción de alternativas metodológicas que incluyen los procesos y prácticas sociales relacionadas a la salud; combinando de manera competente, abordajes cualitativos y cuantitativos en una única estrategia. Al referise a los estudios interculturales en salud Rita de Cássia P. Fernández (2003) hace notar que la epidemiología clásica ha dejado de lado en la construcción del saber epidemiológico, la percepción del sujeto. En este caso de los actores sociales indígenas con relación al proceso salud / enfermedad / atención .

Los programas / proyectos de extensión, desarrollados por Facultades de Odontología de Universidades de distintas regiones del país delimitan, por lo general, para sus prácticas profesionales, comunidades indígenas alejadas de los centros urbanos. De tal manera ponen en contacto a docentes y alumnos con pueblos insertos en contextos históricos- socioculturales de opresión y explotación.

Estos programas iniciales contemplan en sus acciones las siguientes intervenciones: a) revisión Odontológica

b) revelado de Placa bacteriana.

c) extracciones.

d) enseñanza del cepillado.

Se excluye, de este modo, poblaciones adultas con necesidades de tratamientos más complejos (tratamientos de segundo y tercer nivel, prótesis dentales etc.); iniciando a docentes y alumnos en una supuesta relación de "compromiso". En la medida que contactan con población con grandes demandas de atención odontológica las expectativas así creadas refieren para su concreción a responsabilidades institucionales / financieras que contemplen respuestas sanitarias saludables.

En otro plano, las diferencias observadas en las prácticas diarias de autocuidados / automedicación, (alimentación, curación etc), llevadas a cabo por los miembros de estas comunidades; como así también sus particulares modos de transitar el proceso salud / enfermedad / atención conllevan a reflexiones que podemos sistematizar en dos niveles:

Un primer nivel: refiere a una etapa de desarrollo de conocimientos críticos. En este sentido, para abordar el campo de la salud se debe considerar diferentes enfoques que permitan explicarlo y entenderlo. Los aspectos históricos, contextuales, las dimensiones estructurales de relaciones, regularidades, singularidades y las intervenciones de los sujetos y sus interpretaciones en la producción de la realidad son de suma importancia para tratar asuntos complejos de la salud. Deberíamos sustentar un pensamiento crítico (en relación con el modelo médico hegemónico). Aportando conocimientos conceptuales metodológicos / técnicos desde campos científicos biológicos sociales.

Se debe lograr un trabajo en común entre epidemiólogos y cientistas sociales en colaboración mutua y en el intento de construir abordajes y resultados interdisciplinarios ${ }^{2}$ y no meramente multidisciplinares.

Un segundo nivel: ubicado ya en el domino de las prácticas de extensión, de educación y asistenciales debe lograr la conformación de equipos interdisciplinarios de asistencia profesional. Que puedan poner en acción / práctica profesional los aspectos conceptuales /

\footnotetext{
${ }^{2}$ Nos referimos a la construcción de un núcleo de convergencia disciplinar a partir de la integración de distintos niveles teórico-metodológicos provenientes de zonas fronterizas de distintas disciplinas.
} 
técnicos. Así constituido, el grupo de intervención, y ya situado en las respectivas experiencias de campo, el contacto de profesionales con los miembros de la comunidad objeto de interés refiere a lo subjetivo y grupal e incluye confrontaciones con los saberes disciplinarios y de los profesionales miembros del equipo.

Se debaten aquí una amplia gama de consideraciones al momento de efectivizar las acciones tales como:

a) conocimiento de las organizaciones indígenas y de sus reivindicaciones en materia de derechos cívicos en salud.

b) reconocimiento de las actividades comprendidas en los procesos de desarrollo autogestivo por parte de la comunidad. Tales como: pequeños emprendimientos de artesanías, cría de animales. formación de cooperativas a nivel regional etc.

c) reconocimiento, en el marco de políticas nacionales, provinciales y de medios de comunicación, del espacio público donde estas minorías, en sus organizaciones indígenas, se expresan. Y la adaptación de dichos recursos para la transmisión / enseñanza de prácticas preventivas en salud y salud bucal.

d) Contacto con organizaciones locales con proyección nacional. Valga como ejemplo el Encuentro Nacional de la Mujer. De esta manera se logrará la realización en conjunto de acciones de difusión / educación preventiva sanitaria. En un plano operativo.

e) La necesaria utilización de medios técnicos para el registro de datos (grabador, filmadoras, etc.) y modalidades de convivencia. Como así también en las prácticas asistenciales. Deben considerarse legislaciones y protocolos nacionales / internacionales que contemplen valores éticos tales como los derechos de las personas y la salvaguarda de su dignidad e integridad.

\section{Contexto general}

Una revisión de la literatura específica de los campos intervinientes que contemplen la cuestión indígena indica una escasa presencia de estudios epidemiológicos que den cuenta del estado de salud bucal de las poblaciones indígenas en Latinoamérica en general y en Argentina en particular.

En el campo de la salud Pública Odontológica, estos estudios utilizan diferentes indicadores, como son los índices CEO, CPOD, y otros. cuyo propósito es el de describir en, poblaciones específicas, su perfil epidemiológico. Si bien este procedimiento constituye un importante aporte al conocimiento de la situación epidemiológica de estas poblaciones, no agotan a nuestro entender otros aspectos de importancia. Tales como, participación y desarrollos comunitarios y las prácticas autogestivas en salud Azcona S, y Caramés, R. (2006).

Desde esta perspectiva resultan relevantes los estudios que consideran la salud / enfermedad / atención dentro de procesos más amplios que incluyen las redes sociales y familiares que organizan la comunidad, y el estudio de su vinculación con los servicios de salud Freidson, (1978), Menéndez (1990), Infante (1998).

Este enfoque adquiere especial importancia para el estudio de los grupos sociales delimitados en nuestras investigaciones: los qom (tobas), los que por tratarse de una comunidad que presenta rasgos distintivos en su forma de estructuración de lo cotidiano y en su relación con los servicios de salud, con los que interactúa, no resultan ajenos a las fricciones interétnicas entre los prestadores de los servicios y miembros de la población que demandan atención médica / odontológica. Azcona, S y Caramés, R. (2003).

En este contexto los grupos tobas soportan el peso de su condición de migrantes y de indígenas. Las actitudes discriminatorias hacia ellos, por parte de diversos sectores de la 
sociedad local / regional, se ponen de manifiesto en diferentes esferas: principalmente en educación, salud y mercado de trabajo.

$\mathrm{Si}$ esta población indígena cuenta con una legislación nacional y las leyes provinciales que atienden a derechos específicos, en tanto grupo étnicamente diferenciado, la ineficiencia de las políticas puntuales que apuntalen sus enunciados tales como: acceso a la tierra, planes de educación bilingüe e intercultural, programas de salud que contemplen la variable étnica, reconocimiento jurídico de la pluralidad cultural impiden la habilitación de espacios efectivos de vigencia de aquellos derechos que en la actualidad cuentan con un reconocimiento internacional.

Muchas de las familias tobas de las que nos ocupamos migraron recientemente. Otras llegaron al asentamiento en los años 90. En el ámbito de la ciudad de Rosario se produjeron una sucesión de migraciones de grupos domésticos qom siendo las más importantes en los años: 1968, 1987, 1992 y 1994. Durante los años 2003 y 2004 el asentamiento los "Pumitas"de la ciudad de Rosario creció de 150 grupos domésticos a unos 500, aproximadamente. Estos grupos viajan con frecuencia a sus lugares de origen (Chaco y Formosa) y reciben a parientes interesados en la atención médica pública que brinda la ciudad de Rosario. Constituyendo un conglomerado con necesidades sociales acumuladas y población desocupada con precariedad laboral Azcona, S. (2003).

La población Toba asentada en Los Pumitas sustenta concepciones de la salud / enfermedad propias que se evidencian en sus sistemas etiológicos y terapéuticos. Estos sistemas se encuentran en un proceso constante de interacción, intercambio y adopción de concepciones y prácticas provenientes de la biomedicina.

\section{Contexto de investigación.}

Los resultados cualitativos que exponemos se derivan de las investigaciones realizadas en el contexto de los proyectos de investigación SCYT. UNR. Años 2002 y 2005. Durante el proceso de Trabajo de Campo fueron entrevistados interlocutores de la etnia toba asentados en el barrio "Los Pumitas" miembros del "Centro Comunitario QADHUOQTE" y miembros de La Comisión de Salud "NA'M Q'OM", e interlocutores no-tobas de la sociedad local / regional. Conviene destacar aquí:

Que como logro de las movilizaciones sociales en el asentamiento qom del barrio Los Pumitas se ha constituido la organización: "COMUNIDAD QADHUOQTE", con Personería Jurídica en el Registro Nacional de Comunidades Indígenas desde 2004. Entre los objetivos principales que figuran en su estatuto se destacan los siguientes: reafirmación y reivindicación de la identidad y la tradición indígena de la comunidad; defensa de las tierras de la comunidad y reclamo de títulos territoriales aptos para las explotaciones y actividades que sirvan al progreso de las mismas; mejoramiento de las viviendas; actividades de capacitación y formación que sirvan al desarrollo integral de la comunidad.

La comisión de salud "NA'M Q'OM", "surge en el año 2006 y está conformada por miembros de la comunidad toba y miembros de la sociedad local / regional ( trabajadores del Centro de Salud Juana Azurduy, trabajadores sociales y estudiantes de diferentes carreras de la UNR).

Organizada con criterios de participación comunitaria llevan a cabo reuniones estructuradas en torno a inquietudes y necesidades explicitadas por la comunidad. Que podríamos sintetizar así: 1.- Reafirmación y reivindicación de la identidad étnica toba.

2.- Defensa de las tierras comunitarias,

3.-Mejoramiento de la educación (con propuestas de una educación bilingüe). 
4- Salud, en la que se incluye salud bucal.

Un importante número de encuentros giraron en torno al tema salud. En su tratamiento se consideraron actitudes discriminatorias, por parte de la sociedad local / regional / medicina institucionalizada. Expresadas en dificultades para acceder a los servicios de atención localizados fuera de la zona de influencia del barrio (Centros de salud de mediana y alta complejidad). Se explicitó, además, la poca y mala información que los dispensarios locales tenían sobre el proceso de salud / enfermedad / atención con relación a las características propias de la cultura toba y de las pautas de interacción social que ellas generaban.

Es de destacar la organicidad de estas comisiones. Afirman su prestigio en el reconocimiento de su comunidad y emprenden acciones para lograr la representatividad de la misma. Concurren a las reuniones con regularidad alternándose en su participación. Intentan lograr sustentabilidad para sus acciones. Para ello se constituyó un fondo de dinero con el propósito de realizar traslados médicos y satisfacer distintas necesidades de salud como la compra de medicación. Solicitan subsidios para capacitarse como promotores comunitarios de salud. Manifiestan problemas en relación con la comunicación (por sus características lingüísticas) y deseos de establecer sus propios medios de comunicación (solicitan tener una radio de la comunidad etc.).

Mediante procesos cada vez más complejos de transacciones y acuerdos con la Medicina / preventiva / clínica hegemónica, realizan acciones compartidas con los profesionales médicos en un nivel organizativo. Esto es: vacunación, charlas sobre temas preventivos, capacitaciones esporádicas en el Centro de Salud acompañamientos a vecinos para ser tratados en los servicios médicos/odontológicos. Del mismo modo, interactúan con funcionarios de la municipalidad y la provincia con el propósito de obtener insumos para la posta sanitaria y en relación a algunos problemas que enfrenta la comunidad, entre ellos, el alcoholismo y la discriminación

El encuentro que genera este espacio de labor comunitaria, en el marco de los modelos que sustentan las políticas públicas de salud, ha conformado fuertes procesos autogestivos. Caracterizados por la reestructuración de vínculos, la construcción de redes, encuentros, acuerdos, negociaciones y alianzas que han fomentado acciones sociales y culturales. Una expresión de estos movimientos son las prácticas de auto-atención existentes en la comunidad

Como afirma Menéndez, E. (1992), y lo comprobamos en el campo, los procesos autogestionarios en los cuales se inscribe la autoatención permiten a los miembros de la comunidad retener y afianzar los conocimientos propios de su cultura. Dichos procesos permiten la construcción de micropoderes que refuerzan su autonomía en relación con diferentes requerimientos. En nuestro caso, las propuestas sanitarias de los servicios de salud biomédicos locales / regionales.

El campo de negociación así instalado se torna posible en la medida que se aplique una política social integral que fomente el desarrollo de las potencialidades participativas a través de las organizaciones reconocidas por la comunidad.

Las prácticas de automedicación, desarrolladas por los miembros de la comunidad, son consideradas al interior de las prácticas de autoatención. Se vinculan con la atención odontológica, servicios / prestaciones profesionales, y al interior de sus redes de intercambio. De esta manera, pudimos describir las estrategias que sustentan las prácticas de automedicación en los grupos domésticos estudiados. Nos encontramos con una población con características correspondiente a zonas de pobreza urbana. La concurrencia a los efectores de salud está vinculada en su mayoría con la búsqueda de medicamentos (principalmente analgésicos). Los mismos se obtienen sin prescripción odontológica. Es importante la figura del agente sanitario en la obtención del medicamento de patentes. Las enfermedades bucales, se vinculan a la 
dificultad de acceso a tratamientos costosos (prótesis dentales etc,). Tienen temor a no poder reponer las piezas dentales perdidas por prácticas de exodoncias.

Esta situación genera una reafirmación de las prácticas etnomédicas, nos referimos a la aplicación tópica de distintas sustancias u hojas, en algunos casos se colocan directamente en la zona de dolor, aunque también se utilizan medicamentos de patente. Estas acciones se producen tanto en adultos (el 38\% no concurre a ningún efector de salud), como en niños (el $41 \%$ no concurre a servicios asistenciales). Además con frecuencia los adultos realizan las extracciones (en sí mismo, o en los niños). Los niños generalmente no son asistidos odontológicamente, ya que según sus padres no presentan dolor, siendo escasa la atención odontológica específica por considerar que la dentición de este grupo, al ser temporaria: no requiere tratamiento ni control.

Puede comprobarse la relación existente entre condiciones socio económicas y accesibilidad a los servicios con la práctica herbolaria.

Los miembros de la comunidad reconocen las dificultades en el acceso a la atención médica y odontológica. En la medida que los Servicios de Atención Primaria, no cuentan con prestaciones complejas, especialmente odontológicas, deben trasladarse a los hospitales del centro de la ciudad de Rosario. Estas limitaciones en la atención, junto con la drogadicción en los jóvenes y adolescentes (inhalación de la bolsita de Poxirrán, el alcoholismo), conforman una situación de revalorización de sus "remedios" y curadores,

De tal modo la biomedicina /etnomedicina toba, no se restringe a la cultura toba y a los agentes sanitarios a los que asisten. La práctica clínica no está circunscripta al mundo de la racionalidad científica, ella es un producto de fuerzas político- económicas y de las negociaciones de poder que son parte del funcionamiento cotidiano de los servicios de salud. Las políticas públicas actuales están enmarcadas por la retórica de la participación: referida a un proceso conocido por control social. Es decir, la actuación de la sociedad civil en la gestión de políticas públicas en el sentido de controlarlas para que atiendan las demandas y los intereses de la comunidad.

A partir de lo expuesto y en relación con nuestra investigación Cecyt, UNR (2009) (adoptamos un enfoque interdisciplinario, que incluye perspectivas biológicas/ sociales). Se delimitaron Grupos Domésticos Qom Tobas, en ámbitos de pobreza urbana, barrio los "Pumitas" de la ciudad de Rosario. De este modo intentamos detectar los patrones de comportamiento de la población en estudio, en torno a los movimientos de reivindicación en salud / salud bucal / condiciones epidemiológicas del barrio y en respuesta de las políticas sanitarias emanadas de jurisdicciones locales / regionales.

Las entrevistas a miembros Del Centro Comunitario QADHUOQTE y de la Comisión de Salud "NA""MQ"“OM" demuestra que persisten en la población estudiada características de vulnerabilidad socio epidemiológica. Comparten con los grupos indígenas americanos las características de ser los sectores sociales que presentan las tasas más altas de mortalidad, y las más bajas en cuanto a la expectativa de vida. Si bien disminuyeron estos porcentajes, hay acuerdo entre los organismos internacionales / nacionales que la situación es altamente preocupante. No obstante las respuestas sanitarias médico / odontológicas usuales no consideran la consulta y participación efectiva de la comunidad. Las propuestas (Centros de salud) son, para nuestra especificidad (salud / bucal), prácticas de prevención, evaluando las prácticas de cepillado, y la asistencia y percepción de la calidad de los servicios odontológicos.

De tal modo el proceso salud / enfermedad / atención opera dentro de diferentes tipos de relaciones, y especialmente dentro de las relaciones de hegemonía / subalternidad generadas entre los diferentes actores sociales que transaccionan sus distintos saberes.

Desde esta perspectiva, la descripción y análisis de los procesos de salud / enfermedad / atención son referidos a las condiciones de desigualdad socioeconómica, a la diversidad 
sociocultural y a los saberes que operan en términos relacionales entre los diferentes actores sociales que tienen que ver con dicho proceso.

\section{Conclusiones}

Queda claro entonces la necesidad, para este tipo de investigación, de la utilización de un abordaje etnoepidemiológico y la necesidad de la articulación de una estrategia metodológica cuanti-cualitativa.

La vitalidad de prácticas de autoatención puede vincularse a las dificultades con las que se enfrentan a la propuesta sanitaria local / regional. Relacionadas con aspectos socioeconómicos: costo de transporte, por la larga distancia de los servicios de salud de segundo y tercer nivel, y asociados a las estructuras de salud. Nos referimos a la verticalización de las acciones de salud, burocracias y discursos médicos, supuestos o reales prejuicios del personal de salud en relación a los aborígenes y su cultura o relacionados a la propia historia de contacto entre los profesionales y el grupo indígena.

Por otro lado, a partir de la organización autogestionaria la comunidad encuentra una forma para representarse y construir sus propios procesos de salud / enfermedad / atención, en un contexto de rechazo / integración selectivo con relación a las prácticas de la medicina oficial y los valores culturales del propio grupo.

Las demandas en salud, entonces, se realizan desde perspectivas etnopolíticas (derechos jurídicos / salud / educación de los pueblos originarios) articuladas a la realidad del plano local. Esto es a los acuerdos establecidos entre: los servicios de salud / y el Centro Comunitario QADUOHQTE. En el contexto de políticas sociales / sanitarias emanadas de jurisdicciones locales provinciales.

Bibliografía

AMARAL, S.; C. STOKES, S. (Comp.). (2005) Democracia Local. Clientelismo, capital social e innovación política en la Argentina. Buenos Aires, EDUNTREF Editorial De La Universidad Nacional de Tres de Febrero

AZCONA, M. S. (1999). Radovich, J.C; Balazote, A. (comp.).Estudios Antropológicos sobre la cuestión indígena en la Argentina. La Plata. Editorial Minerva.

AZCONA, MS. (2002).Modelo de Atención Odontológico en la Salud Pública Municipal. UNR. Editora.

AZCONA, MS (2008) "Modelo de atención y práctica odontológica en el Hospital Escuela de la Facultad de Odontología de la universidad Nacional de Rosario. Modalidades de acceso y utilización de los servicios según las características socio-culturales de la población local. "C.I.C.E.A. EDITORA. Facultad de Humanidades y Artes U.N.R. ISBN: n 978-950-673-7177. pag. 262.

AZCONA, M. S; ABT, A; CARAMÉS, R. (2003)."Prácticas de autoatención en un contexto de relaciones interétnicas: La situación de los indígenas tobas en el asentamiento los Pumitas de la ciudad de Rosario". Papeles de Trabajo (CICEA) UNR. No 11 pp.135 I.S.S.N. 0327-6147.

AZCONA, M. S.; CARAMÉS, R. (2002) "Modalidades en el consumo de medicamentos por parte de la población asistida en los servicios odontológicos de la FOR”. Papeles de Trabajo. CICEA. UNR. No3 pp. 145-160. I.S.S.N. 0327-6147. 
AZCONA, M. S.; CARAMÉS, R. (2006). "Aproximación a la problemática de la asistencia Odontológica en zonas de pobreza urbana. Análisis de un caso tobas del qom del barrio los Pumitas de la ciudad de Rosario" Papeles de Trabajo. CICEA. UNR. Nº14 pp. 171-191. I.S.S.N. 0327-6147.

BATALLA B, G. "El etnodesarrollo: sus premisas jurídicas, políticas y de organización". Mexico, En obras escogidas de Guillermo B. Batalla, Tomo 2 (pp.464 -480), 1995.

BELLO, A. (2004) Consultor División de Desarrollo Social. Comisión Económica para América Latina y el Caribe. (CEPAL)Santiago de Chile. Libros de la CEPAL

BIGOT, M. (2007). Los aborígenes Qom en Rosario. UNR. Editora.

BORDONI, N. y Col. (1993). "Modelo Alternativo para la atención en salud bucal".Un documento para la transferencia tecnológica". F.O.U.B.A. Fundación N. K. Kellow.

CARDOSO DE OLIVEIRA, R. (2005). "Identidade étnica, reconhecimento e o mundo moral." In : Antropológicas, año 9, v.16 (2) p. 9-40. Recife,

CARVALHO SANTOS, R(2006) "Saúde todo dia. Uma construção coletiva". São Paulo, Editora Hucitec,

CASTRO LUCIC, M. 2008 "La Universalización de la Condición Indígena". México Revista Alteridades, Departamento de Antropología de la Universidad Autónoma Metropolitana . Iztapalapa,

DE SOUSA CAMPOS, G. W. (1998). "Análisis crítico de las contribuciones del cuerpo teórico de la salud pública a las prácticas sanitarias”. Cuadernos Médicos Sociales. No 74 pp. 79-20

FERNÁNDES P.C;R. , (2003) "Uma leitura sobrea a perspectiva etnoepidemiológica". Ciênc. saúde coletiva vol.8 no.3 São Paulo.

FLEURY, S.; OUVERNEY M.A. (2007)."Gestao de Redes. A estratégia de regionalizaçao da saúde". Rio de Janeiro, Editora FGV,.

GERSCHMAN, S; WERNECK VIANA, M.L. (1997) A Miragem da Pós-Modernidade Democracia e Politicas Sociais no contexto da Globalização. Rio de Janeiro, Editora FIOCRUZ

JELIN, E. (2004). "Salud y Migración Regional. Ciudadanía, discriminación y comunicación intercultural". IDES (Instituto de Desarrollo Económico y Social).

MENÉNDEZ, E. (1992)."Grupo Doméstico y proceso salud/enfermedad/atención."Del teoricismo al movimiento continuo". Cuadernos Médicos sociales. $\mathrm{N}^{\mathrm{o}} 59$ pp. 3-18. CESS, Rosario, Argentina.

MENÉNDEZ, E.; SPINELLI, H. (Coord.). “Participación Social ¿Para qué?”. Buenos Aires, Lugar Editorial, 2006.Minayo, S.M.C. "Interdisciplinariedad y Pensamiento Complejo en el Área de la Salud". Salud Colectiva, Enero-Abril, año/vol.4, número 
PÉREZ ARIAS, H. 2007. “ ¿ Autonomía o Desaparición? Pueblos Indígenas en Sudamérica: Chile y Bolivia”. CESLA (Centro de Estudios Latinoamericanos), Universidad de Varsovia.

SPINELli, H. (2004). Salud Colectiva. Cultura, Instituciones y Subjetividad Epidemiología, Gestión y Políticas . Buenos Aires, Lugar Editorial,

VAN DIJK, T.A. (2003).Dominación étnica y racismo discursivo en España y América Latina. Barcelona, Editorial Gedisa, S.A.,

VÁZQUEZ, H. (2002). "Antropología emancipadora y reivindicaciones indígenas". Papeles de Trabajo. UNR.. $\mathrm{N}^{\text {a }} 10$ pp. 26-48. UNR I.S.S.N. 0327-6147.

VÁZQUEZ, H. (2003). "Multiculturalidad, pluriculturalidad y discriminación étnica en Giovanni Sartori y Jurgen Habermas. Reflexiones desde una Antropología latinoamericana crítica y emancipadora". Papeles de Trabajo. UNR. 2003.N ${ }^{\mathrm{a}} 11$ pp. 83-107. I.S.S.N. 03276147.

VÁZQUEZ, H. (2004). Antropología Emancipadora, Derechos Humanos y Pluriculturalidad. Rosario, Homo Sapiens Editora.

VÁZQUEZ, H. (2005). "Dinámica de los procesos étnicos políticos de los tobas asentados en la zona Norte de la ciudad de Rosario, Argentina". Papeles de Trabajo. UNR. 2005. N 13 pp. 5969.

WACQUANT, Loic. (2007) Los Condenados de la Ciudad. Gueto, periferias y Estado. Buenos Aires, Siglo XXI Editores Argentina. 